\title{
Sialic acid histochemistry and the adenoma-carcinoma sequence in colorectum
}

\author{
S Agawa, J R Jass
}

\begin{abstract}
A change in sialic acid, notably loss of 0 acetyl substitution, was studied histochemically in a series of 93 colorectal adenomas that included 14 malignant polyps. Changes in sialic acid were uncommon in adenomas showing mild dysplasia but became increasingly common in moderately and severely dysplastic adenomas and were always present in carcinomatous areas. There was no correlation between a change in sialic acid and either size or villous architecture of benign adenomas. As mucin change often preceded the development of invasive cancer, it could not be used on its own to distinguish between epithelial displacement (pseudoinvasion) and malignant invasion. An abundant luminal accumulation of altered sialic acid in association with an epithelial lining that was non-mucin secreting, however, was specific for cancer. Conversely, the presence of unchanged intracellular or intraluminal O-acetyl sialic acid may help to exclude a diagnosis of malignancy in equivocal cases. The change is not specific for colorectal neoplasms and probably represents a metaplasia to a small intestinal phenotype.
\end{abstract}

It is generally accepted that most, if not all, colorectal adenocarcinomas arise within preexisting adenomas. ${ }^{12}$ It is also clear, however, that adenomas are common lesions and most will not transform into carcinomas. Malignant change in an adenoma shows an important association with size, type (presence of villous architecture), and grade of epithelial dysplasia. ${ }^{1}$ Of these features, grade of epithelial dysplasia is considered to be the most selective risk factor. ${ }^{3}$ Although the last is difficult to prove, indirect evidence now comes from studies of DNA content ${ }^{4}$ and identification of mutations by molecular biological methods. These studies have shown correlations between cumulative genetic errors and grade of epithelial dysplasia, as determined by routine histopathological examination. This information is of limited value for the individual patient (although it might be supposed that a patient harbouring an adenoma showing severe dysplasia would be at increased risk of producing further adenomas). Knowledge of the stage of evolution of an adenoma, however, is important in comparative epidemiological surveys and in increasing our under- standing of the basic mechanisms underlying the adenoma-carcinoma sequence. Of particular interest are the structural, functional, and genetic changes that underlie the conversion of benign adenoma to invasive carcinoma.

There have been relatively few mucin histochemical studies of colorectal adenomas. The reduction of mucin staining that accompanies increasing epithelial dysplasia is well known and has been reaffirmed in a recent detailed study. ${ }^{6}$ Use of the high iron diamine-alcian blue (HID-AB) technique has not disclosed any change in the brown:blue (sulphomucin: sialomucin) ratio that is correlated with epithelial dysplasia, ${ }^{6}$ but interesting changes, implicating sialic acid (loss of O-acetyl groups) have been described. ${ }^{7}$ Changes in sialic acid may be visualised by a red to blue colour change using the periodic acid-Thionin Schiff/potassium hydroxide/periodic acidSchiff (PAT/KOH/PAS) technique. ${ }^{78}$ An alternative approach is to show acquired negative staining with the periodate-borohydride technique (PB/KOH/PAS) combined (in a serial section) with a positive result with diastase PAS. ${ }^{9}$ Neither of these methods provides full insight into the nature of the qualitative change in mucin histochemistry. Thus there are two explanations for the change. Loss of O-acetyl substituents could be accompanied by the retained expression of $\mathrm{N}$-acetyl sialic acid. Alternatively, there could be increased expression of neutral sugars at the expense of sialic acid.

Using the PAT/KOH/PAS technique, Culling et al described a red to blue conversion in colorectal cancer and in foci of malignant change in adenoma. ${ }^{7}$ Partial change (purple colour) occurred in adenomas, but no correlation with a grade of epithelial dysplasia was evident. Their series, however, was small. Using $\mathrm{PB} / \mathrm{KOH} / \mathrm{PAS}$ in tandem with PAS, Greaves et al were impressed by mucin changes in areas of severe dysplasia in adenomas amounting to "focal carcinoma". Changes in adenomas showing lesser dysplasia were less common and were unrelated to the grade of dysplasia. Eide et al documented partial loss of O-acetyl substitution in moderately dysplastic adenomas and showed that the absence of $\mathrm{O}$-acetyl sialic acid was highly predictive of severe dysplasia. ${ }^{8}$ The preceding observations indicate that a major qualitative change in mucin histochemistry accompanies the adenoma-carcinoma sequence, and this seems to be especially obvious at the critical step of malignant conversion.
Correspondence to: J R Jass

Accepted for publication 1 March 1990 
We have used the mild periodic acid Schiff (mPAS) technique to demonstrate $\mathrm{N}$-acetyl sialomucin in normal and diseased colorectal epithelium. ${ }^{10}{ }^{11}$ It is easier to observe positive staining of an abnormally expressed marker than the reciprocal loss of a marker present in the normal tissue. This is particularly true when a quantitative reduction accompanies, and may impede, the assessment of qualitative change. Our studies of colorectal cancer and of epithelial changes in ulcerative colitis show that loss of $\mathrm{O}$-acetylated sialic acid is accompanied by increased expression of $\mathrm{N}$-acetyl sialic acid. We and others have also uncovered new findings that invite considerable care in the interpretation of mucin histochemical data:

1 The relatively low ratio of sialic acid to neutral sugars in the right colon emphasises the need to compare region by region. ${ }^{10}$

2 Constitutional expression of $\mathrm{N}$-acetyl sialic acid as opposed to $\mathrm{O}$-acetyl sialic acid occurs in about $8 \%$ of the normal population. ${ }^{10} \mathrm{~A}$ change from $\mathrm{O}$ - to $\mathrm{N}$-acetyl sialic acid cannot occur in such patients.

3 Uni-cryptal or oligo-cryptal expression of $\mathrm{N}$-acetyl sialic acid occurs in normal colorectal mucosa. ${ }^{12}$ The basis of this phenomenon is unclear but could be due to a mutation-through allele loss in heterozygous subjects. ${ }^{13}$

4 Conversion of $\mathrm{O}$ - to $\mathrm{N}$-acetyl sialic acid occurs in metaplastic (hyperplastic) polyps and in colitic mucosa showing metaplastic type change. ${ }^{11}$

Observations 3 and 4 provide two contrasting mechanisms to account for changes in sialic acid in adenoma or carcinoma-that is, mutational or through epigenetic reprogramming of developmental commitment. A third possibility could be through degradation of secreted mucins by the action of bacterial glycosidases. In view of the latter it is important to distinguish between intracellular and intraluminal mucus because the mucus may have been degraded. Previous studies have not always heeded the location of epithelial mucins nor have they addressed the aforementioned potentially confounding factors.

The aim of our study was to document the loss of O-acetylated sialic acid in a series of colorectal adenomas by using the specific mild PAS technique in parallel with the longer established PAT/KOH/PAS method. As well as attempting to correlate mucin changes with

Table 1 Histopathological and histochemical features of epithelial displacement ("pseudoinvasion") compared with malignant invasion

\begin{tabular}{ll}
\hline $\begin{array}{l}\text { Benign epithelial displacement } \\
\text { ("pseudoinvasion") }\end{array}$ & True malignant invasion \\
\hline $\begin{array}{l}\text { Resembles overlying epithelium and often falls } \\
\text { short of severe dysplasia }\end{array}$ & $\begin{array}{l}\text { More cytological and architectural } \\
\text { atypia than overlying/adjacent } \\
\text { epithelium with features of } \\
\text { severe dysplasia } \\
\text { Stromal haemosiderin absent } \\
\text { Glands surrounded by desmoplastic } \\
\text { stroma }\end{array}$ \\
$\begin{array}{l}\text { Stromal haemosiderin present } \\
\text { Glands surrounded by lamina propria intracellular mucus } \\
\text { N-acetylated intraluminal mucus in } \\
\text { association with absence of } \\
\text { intracellular mucus }\end{array}$ \\
$\begin{array}{l}\text { Abundant intracellular mucus } \\
\text { O-acetylated intraluminal sialic acid }\end{array}$ & \\
O-acetylated goblet cell sialic acid & \\
\hline
\end{tabular}

type, grade, and size of adenoma we wished to test the usefulness of mucin histochemistry in distinguishing between epithelial displacement and true malignant invasion. The rationale for this undertaking is provided by the three previous reports linking sialic acid change with the advent of carcinomatous transformation. ${ }^{7-9}$ Histological criteria for distinguishing between epithelial displacement ("pseudoinvasion") and true invasion have been published, ${ }^{14}$ but individual cases may pose diagnostic difficulties.

\section{Methods}

One hundred and seven adenomas from 93 patients obtained by either endoscopic polypectomy or surgical resection were studied. All adenomas came from the left colon or rectum to avoid the confounding effect of regional differences in expression of sialic acid. Adenomas without surrounding normal mucosa or less than $1 \mathrm{~cm}$ in diameter were excluded. Relatively large adenomas were included to yield a higher proportion of mild PAS positive cases. Large adenomas are also more likely to contain foci of displaced epithelium. Twenty two advanced carcinomas of the left colon or rectum were also studied.

All tissues had been fixed in $10 \%$ buffered formalin and processed routinely. Serial sections $5 \mu \mathrm{m}$ thick were cut and stained with Ehrlich's haematoxylin and eosin, mild PAS, ${ }^{10}$ and by a modified PAT/KOH/PAS technique $^{7}$ in which $1 \%$ cold Thionin Schiff reagent was prepared by adding $1 \mathrm{~g}$ of thionin, $2.5 \mathrm{~g}$ of sodium metabisulfite, and $4 \mathrm{ml}$ of concentrated $\mathrm{HCl}$ to $100 \mathrm{ml}$ of distilled water. The solution was mixed using a magnetic stirrer. Activated charcoal was added and after shaking for one to two minutes the solution was filtered.

Control tissues were included in each staining run. Normal ileum from a specimen of Crohn's disease served as a positive control for mild PAS and gave a blue reaction with PAT/KOH/PAS. A sigmoid colon sample from a patient with diverticular disease served as a negative control for mild PAS and gave a red reaction product with $\mathrm{PAT} / \mathrm{KOH} / \mathrm{PAS}$.

Sections stained by haematoxylin and eosin were studied by one author (JRJ) and the mucin histochemical stains were studied by the other (SA). Adenomas were classified as showing mild, moderate, or severe dysplasia, the last amounting to carcinoma in situ. Polyps were regarded as malignant when carcinomatous epithelium could be observed crossing the line of the muscularis mucosae into the submucosa. Criteria for distinguishing between epithelial displacement (pseudoinvasion) and true malignant invasion are summarised in table 1.

Mild PAS staining was graded as negative or positive and PAT/KOH/PAS staining was graded as blue (including purple) or red. Staining patterns were graded as no change (negative by mild PAS or red by PAT/KOH/ PAS, or both), or as a change when mild PAS stained positively and there was a blue or 
Table 2 Relation between mucin changeintracellular-and size of benign adenoma

\begin{tabular}{llll}
\hline Size & No change & Focal change & $\begin{array}{l}\text { Mosaic or diffuse } \\
\text { change }\end{array}$ \\
\hline$<2 \mathrm{~cm}$ & 30 & 6 & 20 \\
$>2 \mathrm{~cm}$ & 8 & 6 & 8 \\
\hline
\end{tabular}

Total $\chi^{2}=3 \cdot 8, \mathrm{df}=2, \mathrm{p}=0 \cdot 15$.

Table 3 Relation between mucin changeintraluminal-and size of benign adenoma

\begin{tabular}{llll}
\hline Size & No change & Focal change & $\begin{array}{l}\text { Mosaic or diffuse } \\
\text { change }\end{array}$ \\
\hline$<2 \mathrm{~cm}$ & 27 & 8 & 21 \\
$>2 \mathrm{~cm}$ & 6 & 6 & 10 \\
\hline
\end{tabular}

Total $\chi^{2}=3 \cdot 4, \mathrm{df}=2, \mathrm{p}=0 \cdot 18$

purple colour with PAT/KOH/PAS. Change was defined as focal when only a small number of crypts were involved or as mosaic or diffuse when larger areas or the entire polyp showed a change in mucin staining. Assessment of mucin within goblet cells and in an intraluminal location was performed separately. Statistical evaluation was undertaken using the $\chi^{2}$ test.

\section{Results}

Of 107 adenomas, two gave a blue or purple colour with $\mathrm{PAT} / \mathrm{KOH} / \mathrm{PAS}$ when mPAS staining was negative. Conversely, mPAS staining was positive in five adenomas when there was no corresponding change with PAT/ $\mathrm{KOH} / \mathrm{PAS}$. We elected to record a change from $\mathrm{O}$ - to $\mathrm{N}$-acetyl sialic acid only when there was concordance between the two methods. In 15 adenomas the surrounding normal mucosa gave a positive reaction with $\mathrm{mPAS}$ and stained blue with PAT/KOH/PAS. These specimens came from 13 patients. Thus 13 out of $93(14 \%)$ patients showed diffuse expression of $\mathrm{N}$-acetyl sialic acid as a constitutional feature. It is not possible to study the acquired expression of $\mathrm{N}$ acetyl sialic acid in adenomas from such patients and they were removed from the study. Two of the $22(9 \%)$ cancers showed similar background expression of $\mathrm{N}$-acetyl sialic acid. Analysable data were therefore derived from 78 benign adenomas, 14 malignant adenomas, and 20 advanced carcinomas.

There was no association between a change in mucins (either intracellular or intraluminal) and size of benign adenoma (tables 2 and 3 ) or between mucin change and type of adenoma (tables 4 and 5), though trends were observed for both. There was an association, however, between a change in mucins (both intracellular and intraluminal) with grade of epithelial dysplasia (tables 6 and 7). There was a very close association between the demonstration of

Table 4 Relation between mucin changeintracellular-and type of benign adenoma

\begin{tabular}{llll}
\hline Type & No change & Focal change & $\begin{array}{l}\text { Mosaic or diffuse } \\
\text { change }\end{array}$ \\
\hline $\begin{array}{l}\text { Tubular } \\
\begin{array}{l}\text { Tubulovillous } / \\
\text { villous }\end{array}\end{array}$ & 21 & 2 & 9 \\
\hline Total $\chi^{2}=3 \cdot 4, \mathrm{df}=2, \mathrm{p}=0.18$. & 10 & 19
\end{tabular}

Table 5 Relation between mucin changeintraluminal-and type of benign adenoma

\begin{tabular}{llcc}
\hline Type & No change & Focal change & $\begin{array}{l}\text { Mosaic or diffuse } \\
\text { change }\end{array}$ \\
\hline $\begin{array}{l}\text { Tubular } \\
\begin{array}{l}\text { Tubulovillous/ } \\
\text { villous }\end{array}\end{array}$ & 16 & 3 & 9 \\
\hline Total $\chi^{2}=4 \cdot 2, \mathrm{df}=2, \mathrm{p}=0 \cdot 12$. & 22 \\
\hline
\end{tabular}

intracellular and intraluminal changes in mucin (table 8). There were two instances where an extensive mosaic pattern of intraluminal mucin change occurred without obvious intracellular change. Both cases showed severe dysplasia and on careful inspection tiny droplets of mucin expressing $\mathrm{N}$-acetyl sialic acid could be observed in severely dysplastic cells.

Within the frankly carcinomatous areas of malignant adenomas only seven out of 14 specimens included cells containing sufficient amounts of mucus to be analysable. Six of these showed change (loss of $\mathrm{O}$-acetyl sialic acid). Abundant intraluminal mucin that was strongly positive with mPAS and blue with $\mathrm{PAT} / \mathrm{KOH} / \mathrm{PAS}$, however, was seen in the carcinomatous areas of all 14 malignant polyps. There was therefore a very clear association between neoplastic evolution and intraluminal mucin change. In mild, moderate, and severe dysplasia and in carcinoma intraluminal mucin change (loss of $\mathrm{O}$-acetyl sialic acid) was observed in $8 \%, 40 \%, 86 \%$ and $100 \%$ of cases, respectively. The advanced cancers were similar to the malignant adenomas. Ten of the 20 cancers expressed intracellular mucus (usually very small amounts) and the change could be detected in nine of these. Intraluminal mucus, however, was abundant and showed loss of $\mathrm{O}$-acetyl sialic acid in all 20 cases.

Thirteen adenomas showed a distinct mosaic pattern of mucin staining, some areas being negative with mPAS and red with $\mathrm{PAT} / \mathrm{KOH} /$ PAS, with adjacent areas being strongly positive with mPAS (figs $1 \mathrm{a}$ and $\mathrm{b}$ ) and blue with PAT/KOH/PAS. Despite the correlation between dysplasia and mucin change in this series of adenomas, areas showing changed sialic acid within the mosaic polyps were not obviously more dysplastic than the unchanged areas. In five of the 13 adenomas, however, a

Table 6 Relation between mucin changeintracellular-and grade of epithelial dysplasia

\begin{tabular}{llcc}
\hline $\begin{array}{l}\text { Grade of } \\
\text { dysplasia }\end{array}$ & No change & Focal change & $\begin{array}{l}\text { Mosaic or diffuse } \\
\text { change }\end{array}$ \\
\hline $\begin{array}{l}\text { Mild } \\
\begin{array}{l}\text { Moderate to } \\
\text { severe }\end{array}\end{array}$ & 20 & 2 & 2 \\
\hline
\end{tabular}

Total $\chi^{2}=24 \cdot 2, \mathrm{df}=2, \mathrm{p}=0.0001$.

Table 7 Relation between mucin changeintraluminal-and grade of epithelial dysplasia

\begin{tabular}{llcl}
\hline $\begin{array}{l}\text { Grade of } \\
\text { dysplasia }\end{array}$ & No change & Focal change & $\begin{array}{l}\text { Mosaic or diffust } \\
\text { change }\end{array}$ \\
\hline $\begin{array}{l}\text { Mild } \\
\begin{array}{l}\text { Moderate to } \\
\text { severe }\end{array}\end{array}$ & 20 & 2 & 2 \\
\hline Total $\chi^{2}=18$ & 10 & 26 \\
\hline
\end{tabular}


Table 8 Synchronicity of mucin change in lumen and cells of benign adenomas

\begin{tabular}{llcl}
\hline \multirow{2}{*}{$\begin{array}{l}\text { Changes in } \\
\text { lumen }\end{array}$} & \multicolumn{2}{l}{ Change in cells } & $\begin{array}{l}\text { Mosaic } \\
\text { or diffuse } \\
\text { change }\end{array}$ \\
\cline { 2 - 4 } & No change & Focal change & 0 \\
\hline None & 33 & 0 & 0 \\
Focal & 2 & 12 & 28 \\
Mosaic/diffuse & 2 & 0 & \\
\hline
\end{tabular}

Total $\%^{2}=130 \cdot 4, \mathrm{df}=4, \mathrm{p}=0.0001$.

morphological difference between changed and unchanged areas could be seen. In unchanged areas there seemed to be a single population of goblet cells (fig 2a); in changed areas the goblet cells were round and distended by mucus that was strongly basophilic with Ehrlich's haematoxylin. These cells were bordered by brightly eosinophilic columnar cells, sometimes containing small amounts of apical mucin (fig $2 b$ ). The nuclear changes (enlargement and hyperchromatism) were of a similar magnitude in both areas.

Seven benign adenomas showed pseudoinvasion (fig 3). All seven showed moderate dysplasia and the misplaced epithelium included goblet cells. In three cases the goblet cells and associated intraluminal mucus were positive with mPAS and blue with $\mathrm{PAT} / \mathrm{KOH} /$ PAS. Pools of extracellular mucin, present in four cases, also showed the mucin change.

\section{Discussion}

The results indicate that a qualitative change in the structure of sialic acid (O-to $\mathrm{N}$-acetyl sialic acid) occurs in tandem with increasing grades of epithelial dysplasia within a series of colorectal adenomas. This was a selected series, each adenoma being at least $1 \mathrm{~cm}$ in diameter. This accounts for the high proportion of examples showing moderate to severe dysplasia and may explain why we were able to show a clear difference between adenomas showing mild, moderate, and severe dysplasia when this has been the experience of some ${ }^{8}$ but not others. ${ }^{79}$ Rather, previous studies have emphasised the difference between adenoma and carcinoma or carcinoma in situ (severe dysplasia).$^{79}$ Another factor is the subjectivity of the PAT/KOH/ PAS technique with the inherent difficulties of separating red from red/purple from blue/ purple from blue. Loss of reactivity with the $\mathrm{PAB} / \mathrm{KOH} / \mathrm{PAS}$ method is equally hard to gauge. In contrast, we experienced no difficulty in scoring positive or negative with the mPAS technique. In five cases a change with mPAS was not accompanied by a discernible switch from red to blue with PAT/KOH/PAS. This probably reflects the greater sensitivity of the mPAS method. There was no significant association between mucin change and size or type of adenoma, though trends were evident. This is a little surprising given the correlation that exists between dysplasia, size, and type of adenoma. $^{1}$ If the series had been more representative (through the inclusion of adenomas less than $1 \mathrm{~cm}$ in diameter), however, the trend for size compared with mucin change would probably have become significant.

Despite the preceding observations, it is clear that the relation between mucin type and dysplasia is not absolute. Areas of mild dysplasia may show the change whereas foci of moderate dysplasia may not. This is not simply a reflection of the subjectivity of grading epithelial dysplasia. This fact becomes clearer when the heterogeneity within adenomas is heeded. Thirteen adenomas showed a mosaic pattern with the mild PAS technique. mPAS positive and negative areas within the same adenoma, however, could not be distinguished in terms of architectural pattern or cytology (the main variables used in grading dysplasia). This suggests that although dysplasia and mucin changes are associated phenomena, the mechanisms underlying the process of neoplastic transformation and the process of mucinous change may differ fundamentally. In fact an unexpected finding in the present study suggests that the change in mucin can be explained as part of a change in the developmental commitment of the epithelium - that is, metaplasia. Thus in five of the 13 adenomas showing heterogeneous expression of $\mathrm{N}$-acetyl sialic acid positive and negative areas could be distinguished morphologically. In the mPAS positive areas two cell types were evidentgoblet cells and eosinophilic columnar cells secreting little or no mucus. In mPAS negative areas (that included mucus producing cells)
Figure 1 Junctional area in adenoma showing mosaic type mucin change. $(A)$ is stained with Ehrlich's haematoxylin and shows the goblet cell mucus in the left field to be more basophilic than the mucus in the right field. In (B) ( stained with mild $P A S$ ) the left hand field is positive whereas the right hand field is completely negative.

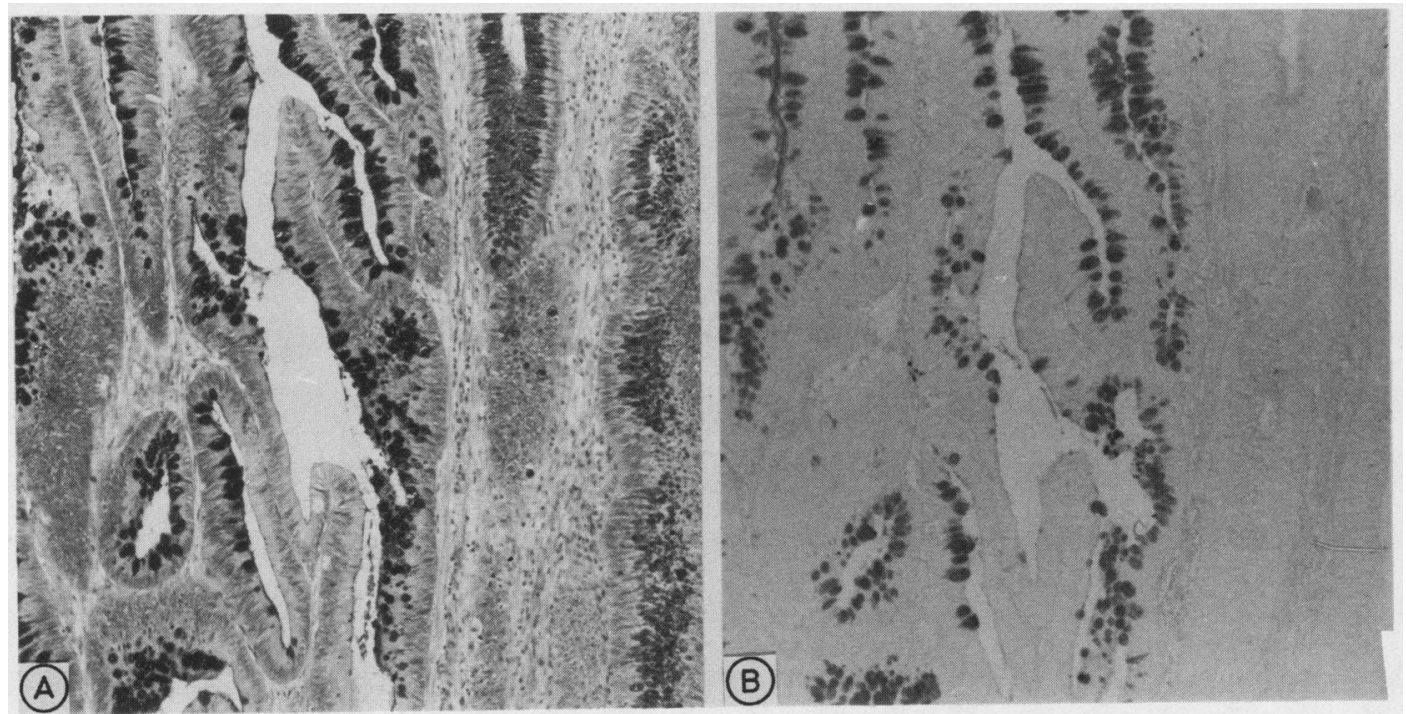


Figure $2(A)$ is from right hand field from fig 1 and $(B)$ is from left hand field. On cytological grounds the two fields show moderate epithelial dysplasia, but on morphological grounds the fields are different. ( $A$ ) shows a single population of mucous cells whereas (B) includes distended goblet cells and $a$ population of eosinophilic columnar cells, some secreting small amounts of apical mucin

(haematoxylin and eosin).

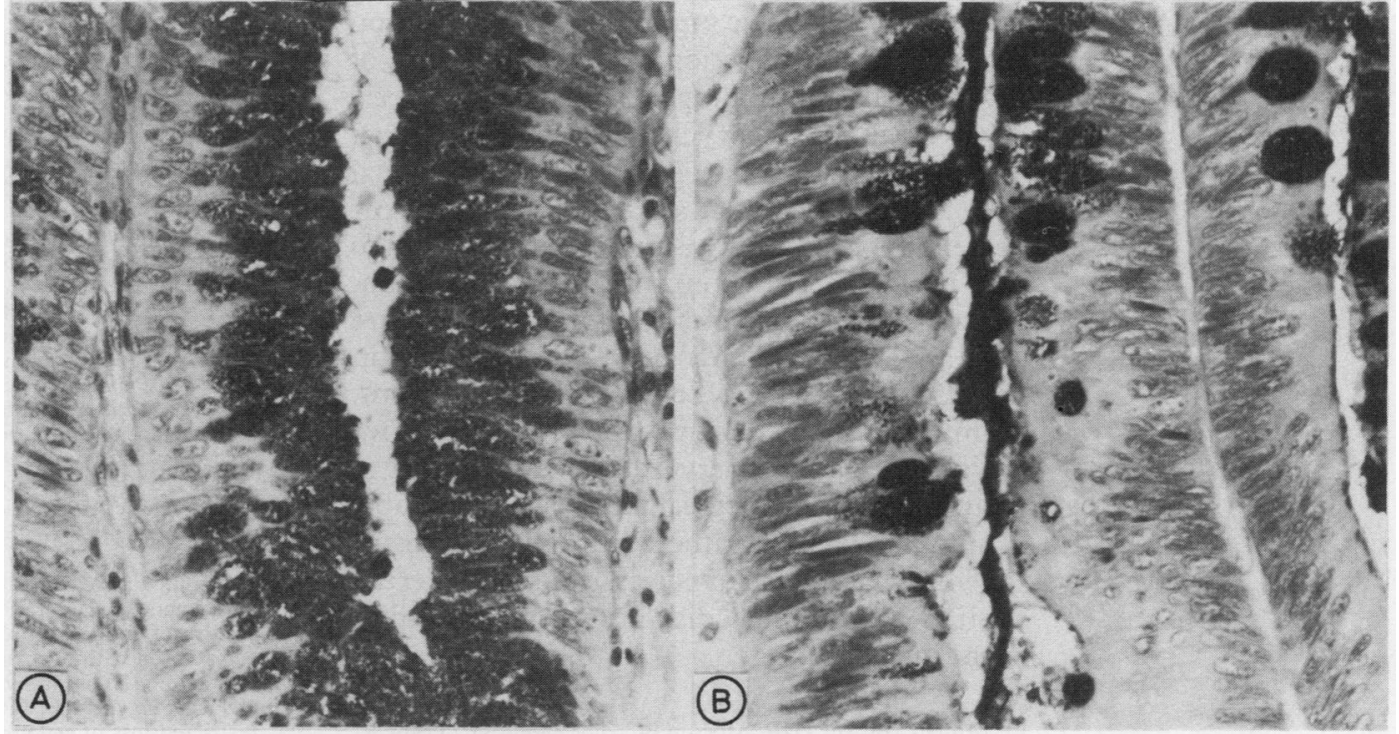

there seemed to be only one cell type-a mucous or goblet cell.

It is tempting to draw morphological and functional comparisons between mPAS positive zones in adenomas and small intestinal epithelium. Small intestinal epithelium comprises columnar absorptive cells and goblet cells secreting, particularly in the proximal small bowel, $\mathrm{N}$-acetyl sialic acid. Evidence of small intestinal metaplasia in colorectal neoplasms can be gleaned from other sources too. ${ }^{15}$ Considering the common occurrence of intestinal metaplasia in gastric neoplasms, there should be no fundamental objection to the possibility of a similar process occurring in colorectal neoplasms. This observation may

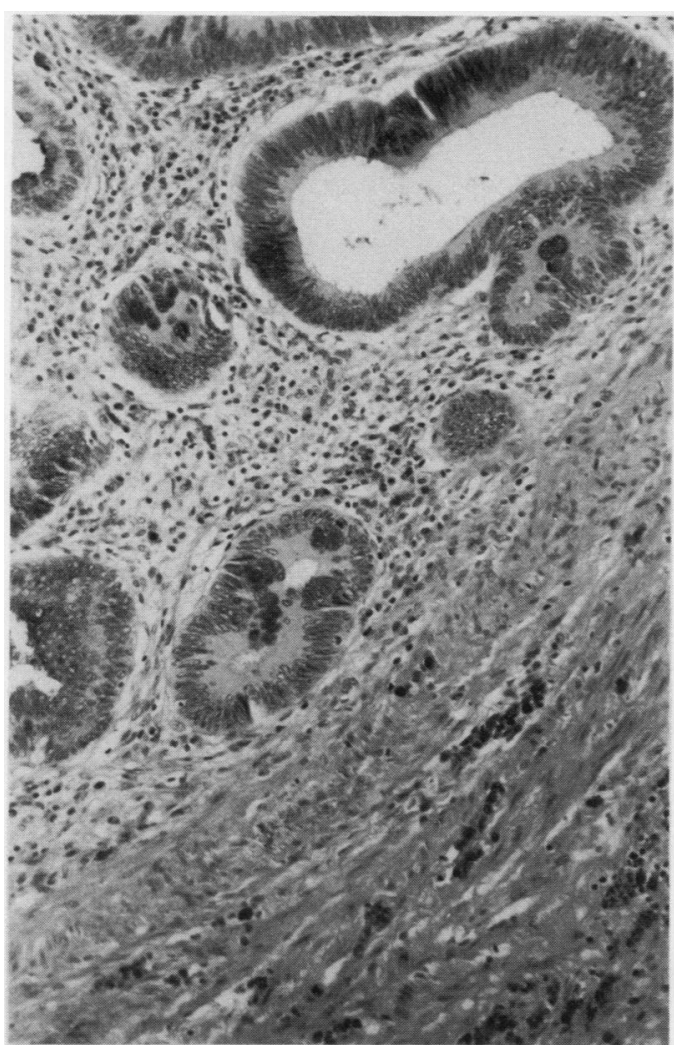

Figure 3 Area of epithelial displacement. Glands show moderate dysplasia and are surrounded by lamina propria. Goblet cells are present and the surrounding stroma includes abundant haemosiderin (haematoxylin and eosin). help to disentangle the primary events leading to neoplasia from changes that are essentially epigenetic or secondary to the basic genetic lesion. Many if not most "tumour associated" changes could be accommodated by the general term metaplasia and hence be regarded as epiphenomena. The secretion of $\mathrm{N}$-acetyl sialic acid by colorectal cancer can be viewed in this light. Given the association between grade of adenomatous dysplasia and changed mucin staining, there is little justification for proposing a separate mechanism for $\mathrm{N}$-acetyl sialic acid expression in malignant epithelium (such as through bacterial degradation of O-acetyl sialic acid). Although colorectal cancer mucin is largely intraluminal, there is an excellent correlation between the intracellular and intraluminal distribution of mucin change. Thus intraluminal mucin seems to retain the histochemical properties of the originating intracellular mucin.

Are mucin changes of clinical value in helping to distinguish simple epithelial displacement and true malignant invasion? In all 14 malignant adenomas the malignant infiltrating epithelium contained intraluminal mucus that stained blue with PAT/KOH/PAS and gave a positive reaction with mPAS ( $\mathrm{N}$-acetyl sialic acid). Goblet cells were present in seven cases but were few in number and secreted small amounts of mucus. In six the mucus expressed $\mathrm{N}$-acetyl sialic acid. Specimens of advanced colorectal cancer showed similar findings. In three of seven polyps showing epithelial displacement intraluminal mucus stained blue with $\mathrm{PAT} / \mathrm{KOH} / \mathrm{PAS}$ and gave a positive reaction with mPAS. Because these examples showed unequivocal pathological evidence of simple displacement, it is clear that changes in expression of sialic acid cannot be regarded as specific for malignant invasion. This result is not surprising, given the high incidence of mucin change in adenomas showing moderate or severe dysplasia. All seven adenomas with epithelial displacement showed moderate dysplasia.

Despite these observations, it is evident that loss of $\mathrm{U}$-acetyl sialic acid is a sensitive marker of malignancy. All malignant lumina contained 
blue/mPAS positive mucin. If this observation is taken in conjunction with the absence of goblet cells the finding is specific for cancer. Conversely, lack of change in intraluminal mucus seems to be specific for benign epithelial displacement. Further studies of well differentiated mucin secreting adenocarcinoma need to be performed to confirm this observation. Extracellular pools of $\mathrm{N}$-acetyl sialomucin are not a feature of malignancy, being present in polyps showing simple epithelial displacement. If mucin histochemistry is applied with care we believe that it may serve as a useful adjunct to routine histopathological interpretation. Table 1 summarises histopathological as well as histochemical criteria that may assist in the differentiation of epithelial displacement and malignant invasion.

1 Muto T, Bussey HJR, Morson BC. The evolution of cancer of the colon and rectum. Cancer 1975;36:2251-76.

2 Jass JR. Do all colorectal carcinomas arise in pre-existing adenomas? World J Surg 1989;13:45-51.

3 Jass JR, Morson BC. Epithelial dysplasia in the gastrointestinal tract. In: Jerzy Glass GB, Sherlock P, eds. Progress in gastroenterology. Vol 4. New York: Grune and Stratton, 1983:345-71.

4 Goh HS, Jass JR. DNA content and the adenoma-carcinoma sequence in the colorectum. J Clin Pathol 1986;39:387-92. Vogelstein B, Fearon ER, Hamilton SR, et al. Genetic alterations during colorectal-tumor development. $N$ Engl J Med 1988;319:525-32.

6 Griffioen G, Bosman FT, Verspaget HW, de Bruin PAF Biemond I, Lamers CBHW. Mucin profiles and potential for malignancy of human colorectal adenomatous polyps. Cancer 1989;63:1587-91.

7 Culling CFA, Reid PE, Clay MG, Dunn WL. A new histochemical technique of use in the interpretation and diagnosis of adenocarcinoma and villous lesions in the large intestine. J Clin Pathol 1977;30:1056-62.

8 Eide TJ, Nielsen K, Solberg S. Dysplasia in colorectal adenomas related to the presence of $\mathrm{O}$-acetylated sialic mucin and to morphometric measurements. Acta Pathol mucin and to morphometric measurements. Acta Pat

9 Greaves P, Filipe MI, Abbas S, Ormerod MG. Sialomucins and carcinoembryonic antigen in the evolution of colorectal cancer. Histopathology 1984;8:825-34.

10 Sugihara K, Jass JR. Colorectal goblet cell sialomucin heterogeneity: its relation to malignant disease. J Clin Pathol 1986;39:1088-95.

11 Jass JR, Sugihara K, Love SB. Basis of sialic acid heterogeneity in ulcerative colitis. J Clin Pathol 1988;41:388-92.

12 Lev R, Lance P, Camara P. Histochemical and morphologic studies of mucosa bordering rectosigmoid carcinomas: comparison with normal, diseased and malignant colonic epithelium. Hum Pathol 1985;16:151-61.

13 Fuller CE, Davies RP, Williams GT, Williams ED. Crypt restricted loss of sialomucin O-acetylation in the human colon-a marker of somatic mutation? J Pathol 1989; 157:174A.

14 Muto T, Bussey HJR, Morson BC. Pseudocarcinomatous invasion in adenomatous polyps of the colon and rectum. J Clin Pathol 1973;26:25-31.

$15 \mathrm{Ma} J$, De Boer WGR, Ward HA, Nairn RC. Another oncofetal antigen in colonic cancer. Br J Cancer 1980; 41:325-8. 